\title{
The peculiar dust properties of the LMC and their implications on modelling SEDs of galaxies
}

\author{
Frédéric Galliano \\ Service d'Astrophysique, CEA/Saclay, L'Orme des Merisiers, 91191 Gif-sur-Yvette \\ email: frederic.galliano@cea.fr
}

\begin{abstract}
In a recent study based on the modelling of spatially resolved Spitzer and Herschel observations of the Large Magellanic Cloud (LMC), we have shown that the infrared (IR) to submillimetre (submm) grain opacities are systematically higher in the LMC than in our Galaxy. This discovery demonstrates an evolution of the dust properties with environment. Here, we discuss the consequences of this evolution on the modelling of SEDs of galaxies, emphasizing that the uncertainties on the grain properties can lead to erroneous interpretations of the structure of the interstellar medium (ISM) of galaxies, if no additional independent constraints are used.
\end{abstract}

Keywords. dust, extinction, galaxies: ISM, Magellanic Clouds

\section{The Dilemma of dust SED modelling: emissivity versus geometry}

The SED modelling of a region large enough to encompass different components of the ISM (H II region, PDR, cirrus, etc.) is degenerate. This degeneracy comes from the fact that, using sparse broadband fluxes and no additional constraints, the SED can be fit with different combinations of macroscopic properties (i.e. the spatial distribution of matter and starlight intensity) and microscopic properties (grain cross-sections, size distributions, etc.). Fig. 1 demonstrates this effect on the integrated SED of $1 / 4$ of the LMC (Galliano et al. 2011).

Dust radiative transfer models are designed to constrain these macroscopic properties, assuming that the microscopic properties are well known. However, there are several reasons pointing toward significant variations of the intrinsic grain properties with the environment. The size distribution could be altered by grain destruction in shocks or grain growth in dense regions. Likewise, the grain composition and abundance are affected by grain coating in molecular clouds or by the evolution of the various sources responsible for the elemental enrichment (SN, AGB, etc.). The latter would imply a systematic evolution of the dust composition, and therefore of the grain cross-sections, with metallicity.

\section{Single black body or 3D radiative transfer, in the Her schel era?}

We have attempted to constrain some of these evolutionary processes by focussing on the high spatial resolution $(\simeq 10 \mathrm{pc})$ Spitzer and Herschel observations of the LMC. Our model is based on realistic grain properties and accounts for a distribution of starlight intensity (Galliano et al. 2011, for more details). When fit to the observations, it provides the dust mass and mean starlight intensity of each pixel. Since we include SPIRE fluxes $(250,350,500 \mu \mathrm{m})$, which are fully determined by the distribution of equilibrium temperature of the big grains, the distribution of starlight intensity is crucial. We have shown that performing single black body fits on these data leads to systematic underestimates of the dust mass (appendix C.2 of Galliano et al. 2011). Moreover, the discrepancy is larger 


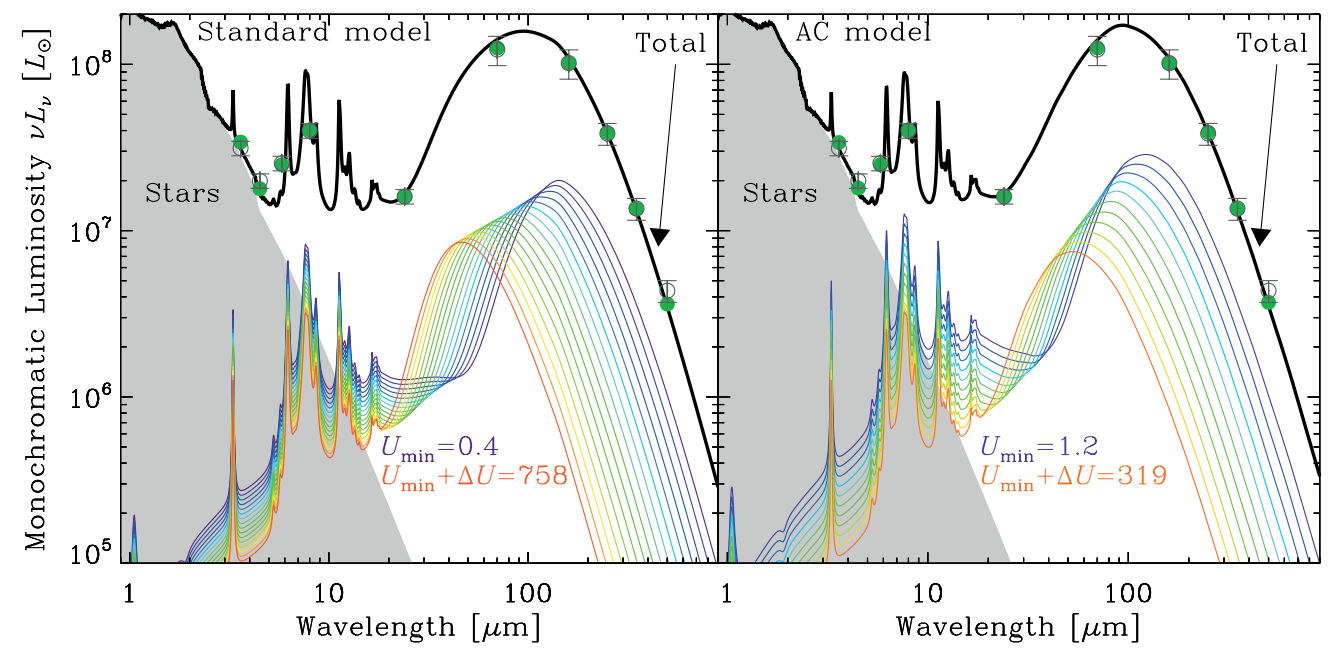

Figure 1. SED model of $1 / 4$ of the LMC with two different grain mixtures (Galliano et al. 2011). For each panel: the gray filled component is a stellar continuum; the SEDs correspond to starlight intensities varying between $U_{\mathrm{min}}$ and $U_{\mathrm{min}}+\Delta U$ in units of $2.2 \times 10^{-5} \mathrm{~W} \mathrm{~m}^{-2}$; the thick black line is the total model; the filled dots are the synthetic photometry; the open circles with error bars are the observations. The standard model is composed of the mixture of PAHs, graphite and silicate satisfying the extinction, emission and depletion constraints for our Galaxy (Zubko et al. 2004). The AC model is the standard model replacing graphite with amorphous carbons. The $A C$ model satisfies depletion constraints but has a higher submm opacity than the standard model. Both models fit equally well the LMC fluxes.

in dense regions, since the gradient of temperature is larger. It means that performing single black body fits biases the trends of dust mass surface density. In particular, it may affect the dust derived $\mathrm{X}(\mathrm{CO})$ factors.

A detailed radiative transfer is not necessary in our case, since our objective is not to derive the 3D structure of the ISM, within each pixel. Having a numerically light model allows us to rigorously propagate the observational uncertainties throughout the entire fitting process (Fig. 2). It allows to account for correlated calibration errors and the effect they have on the non-linearity of the model (Galliano et al. 2011).

\section{The Test case of the Large Magellanic Cloud: enhanced emissivity}

Assuming dust properties of the Milky Way (Zubko et al. 2004, standard model) with this approach leads to gas-to-dust mass ratios lower than the values permitted by the elemental abundances. In other words, Galactic grains applied to the LMC require more heavy elements locked up in dust than what is available in the ISM (Galliano et al. 2011). This model is not physical. We have explored the possbility that a large fraction of the gas could have been overlooked and concluded that, although this effect plays a role, it can not explain this discrepancy. In particular, the gas-to-dust mass ratio is unrealistic, even in the diffuse ISM, where the gas mass is more secure than in the dense ISM.

We have fit an alternate realistic dust composition, which is more emissive (replacing graphite by amorphous carbons; $A C$ model). This composition is consistent with the elemental abundances. In summary, this study demonstrates that the grains in the LMC are systematically more emissive than in the Milky Way, in the submm regime. If this property is the consequence of the different evolutionary stages of the Milky and the LMC $\left(Z \simeq 1 / 2 Z_{\odot}\right)$, then we could expect such a variation of the grain opacities to be 

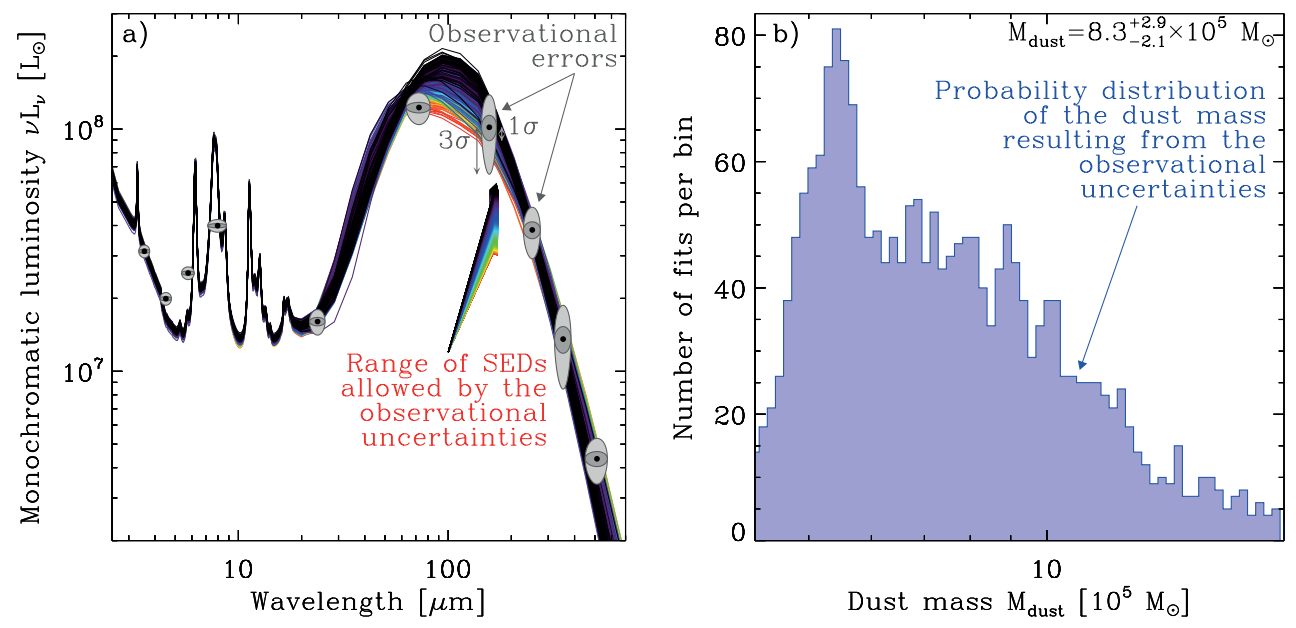

Figure 2. a) Fits of the randomly perturbed SED of Fig. 1. The grey ellipses show the observational uncertainties. The lines are models fit to the randomly perturbed values, taking into account the correlation between the systematic calibration errors. This process is described in details in Galliano et al. (2011). b) Resulting probability distribution of the dust mass for the fits in panel a). It allows us to estimate the uncertainty on the dust mass for several confidence intervals.

enhanced in low-metallicity galaxies. Independent studies suggest this effect is indeed more pronounced in the SMC than in the LMC (Roman-Duval et al. 2011).

\section{Implications: degeneracies of dust SED Models of Galaxies}

Our study has demonstrated that there is a systematic variation of the grain opacities between galaxies. Consequently, modelling the SED of a galaxy with the grain properties of the Milky Way, without additional constraints (gas tracers or detailed geometry, etc.), could lead to gross errors on its derived dust content and ISM structure. For instance, assuming that the grains in this particular galaxy are more emissive than Galactic dust, an excess IR emission excess could be misinterpreted as a population of inactive clumps, while it would simply be the emission from the regular diffuse ISM and PDRs.

There are several ways to tackle this degeneracy, depending on the available data. 1. Observations of the dust emission at high enough spatial resolution can allow to extract a typical SED of the diffuse ISM, assumed isothermal, and constrain its grain emissivity. 2. Direct estimate on the elemental depletion would also provide valuable information on the dust abundance and composition. 3. Extinction (not attenuation) curves can be useful to test different dust mixtures. 4. The total gas content, together with the metallicity, provides estimates of the expected gas-to-dust mass ratio.

\section{References}

Galliano, F., Hony, S., Bernard, J.-P., et al. 2011, A\&A, in press

Roman-Duval, J., Gordon, K. D., Meixner, M., et al. 2011, in AAS Meeting 218, 129.01

Zubko, V., Dwek, E., \& Arendt, R. G. 2004, ApJS, 152, 211 